\title{
Vergleichende Betrachtung verschiedener Methoden zur Bestimmung der Inhomogenität von Thermoelementen
}

\author{
Philipp Germanow ${ }^{1}$, Patrick Mehring ${ }^{1}$, Herbert Neumann ${ }^{1}$, \\ Silke Augustin', Thomas Fröhlich', Helge Mammen ${ }^{2}$ \\ ${ }^{1}$ Reckmann GmbH, Werkzeugstraße 19-23, D-58093 Hagen \\ ${ }^{2}$ TU IImenau, Gustav-Kirchhoff-Str. 1, D-98693 IImenau
}

\section{Zusammenfassung}

Die Homogenität des Thermomaterials nimmt Einfluss auf die Kalibrierfähigkeit und die im Betrieb erreichbare Genauigkeit eines Thermoelementes. Eine Unstetigkeit im Thermomaterial, nachfolgend als Inhomogenität bezeichnet, wird jedoch schon während des regulären Einsatzes des Thermoelementes durch mechanische und thermische Beanspruchungen ausgebildet. Um nun Aussagen über den dadurch entstandenen Messfehler zu treffen, muss die Inhomogenität erfasst werden. Dies wird im Allgemeinen durch zwei unterschiedliche Messverfahren möglich. Im Rahmen der Einbindung einer neuen Messmethode zur Bestimmung der Inhomogenität in einem bestehenden Kalibrierlabor wurden Untersuchungen beider Verfahren vorgenommen. Die daraus entstandenen Versuchsanlagen beider Funktionsprinzipe und die dort aufgenommenen Inhomogenitätsprofile werden vergleichend gegenübergestellt. Abschließend erfolgt eine Einordnung der Messverfahren anhand ihrer Zweckmäßigkeit im bestehenden Kalibrierbetrieb.

Keywords: Thermoelement, Inhomogenität, Ein-Gradienten-Verfahren, Zwei-Gradienten-Verfahren, Messunsicherheitsbeitrag

\section{Entstehung von Inhomogenitäten}

Die Drift eines Thermoelementes, also die Veränderung der erzeugten Thermospannung im Laufe der Betriebszeit, geht stets von einer Änderung des Seebeck-Koeffizienten aus. Letztere sorgt dabei für die Ausprägung von Inhomogenitäten entlang des Thermoelementes. Eine Inhomogenität entsteht beispielsweise durch die Veränderung der chemischen Zusammensetzung, eine Veränderung des metallurgischen Zustandes oder das Einwirken äußerer mechanischer Spannungen [1].

\section{Auswirkung von Inhomogenitäten}

Im Gegensatz zu dem Missverständnis der alten Thermoelement-Theorie resultiert die erzeugte Thermospannung nicht aus der Temperaturdifferenz zwischen Mess- und Vergleichsstelle. Sie entsteht vielmehr durch den Temperaturgradienten und den lokalen Seebeck-Koeffizienten $S(T, x)$ entlang des Thermoelementes [1]:

$$
\mathrm{U}=\int_{0}^{L} S(T, x) \frac{\partial T}{\partial x} d x
$$

Aufgrund dieses Sachverhaltes wirkt sich die Inhomogenität deutlich auf die, in Abhängigkeit von der jeweiligen Einbausituation, erfasste Thermospannung des Sensors aus. Bei einem inhomogenen Thermoelement ist daher die erzeugte Thermospannung verschiedener Einbausituation, beispielsweise während der Kalibrierung und bei Inbetriebnahme, nicht direkt vergleichbar. Der Messfehler, ausgelöst durch die Inhomogenität des Sensors, muss daher zwingend in der Messunsicherheit der Kalibrierung des Sensors berücksichtigt werden.

\section{Erfassung von Inhomogenitäten}

Die Erfassung von Inhomogenitätsprofilen basiert grundsätzlich auf einem bekannten Temperaturgradienten, welcher über das Thermoelement bewegt wird. Der Temperaturgradient resultiert dabei aus dem axialen Temperaturverlauf entlang des zu untersuchenden Sensors. Auf Basis der lokal unterschiedlichen Seebeck-Koeffizienten entlang des Thermoelementes sorgt eine Verschiebung des Temperaturgradienten für eine Veränderung der erzeugten Thermospannung. Wenn die Thermospannung nun an diskreten Punkten entlang des Sensors aufgenommen wird, folgt daraus das 
sogenannte Inhomogenitätsprofil des Sensors. Dabei ist die erfasste Thermospannung ein Maß für die Variation des SeebeckKoeffizienten des Sensors.

Anhand der ausgebildeten Temperaturgradienten kann dabei in Ein-Gradienten- und Zwei-Gradienten-Messverfahren zur Erfassung von Inhomogenitätsprofilen unterschieden werden [2].

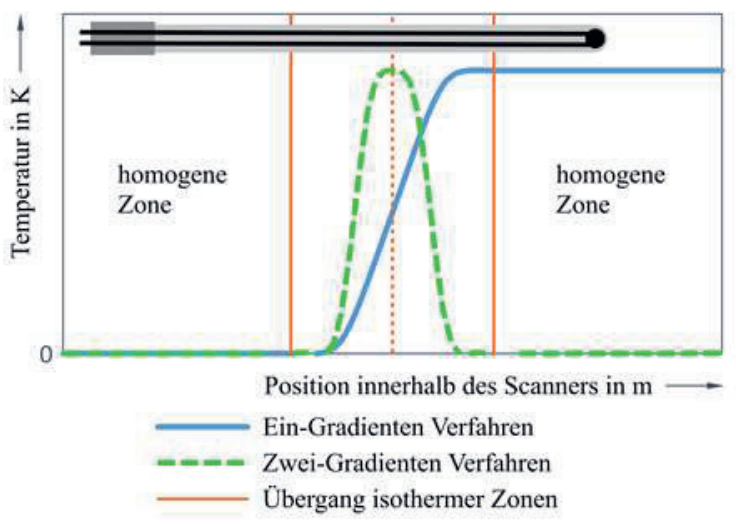

Abb. 1: Schematischer Temperaturverlauf des Ein- und Zwei-Gradienten Verfahrens [3]

Beim Ein-Gradienten-Verfahren wird ein Thermoelement von einer kalten homogenen Zone in eine heiße homogene Zone eingetaucht, beziehungsweise aus ihr herausgezogen. Mit dem Übergang zwischen den homogenen Zonen ergibt sich der in Abb. 1 dargestellte Temperaturverlauf entlang des Thermoelementes.

Im Gegensatz dazu wird beim ZweiGradienten-Verfahren nur eine lokal begrenzte Hitzequelle über das Thermoelement hinweg bewegt. Dabei bleiben die Mess- und die Vergleichsstelle des Sensors auf derselben Temperatur (vgl. Abb. 1).

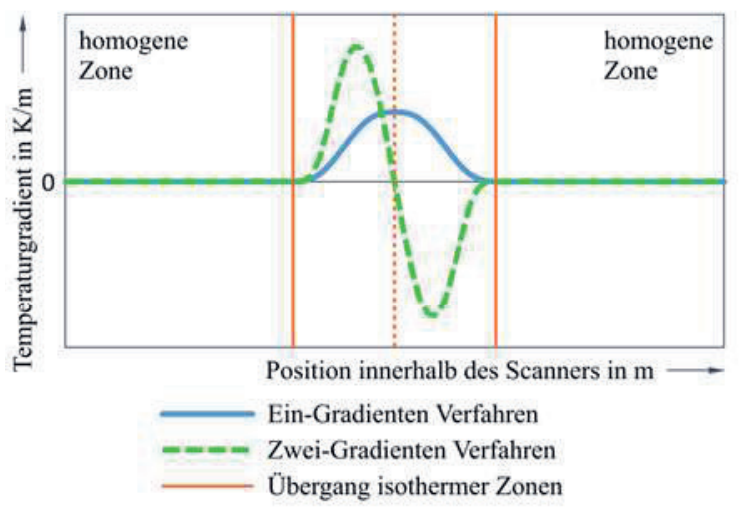

Abb. 2: Schematischer Temperaturgradient des Ein- und Zwei-GradientenVerfahrens [3]
Der Übergang zwischen den isothermen Zonen erfolgt dabei nicht sprunghaft. An den Grenzen der jeweiligen isothermen Zonen beider Messverfahren resultieren stets, die in Abb. 2 dargestellten, Temperaturgradienten. Der Temperaturgradient ist dabei ein Maß dafür, wie schnell die Temperatur entlang des zu untersuchenden Thermoelementes ansteigt. Hier ist jedoch stets darauf zu achten, dass der tatsächlich wirkende Temperaturgradient, auch bei idealer Abgrenzung der homogenen Zonen in der Versuchseinrichtung, stark von der Wärmeleitung entlang des Thermoelementes bestimmt wird [4].

\section{Messungen im Ein-Gradienten-Verfahren}

Die im Ein-Gradienten-Verfahren erfasste Thermospannung resultiert einzig aus dem einfachen Temperaturgradienten zwischen beiden homogenen Zonen. Dementsprechend beschreibt die erfasste Thermospannung hier direkt den innerhalb des Gradienten erfassten Seebeck-Koeffizienten (vgl. Abb. 3).

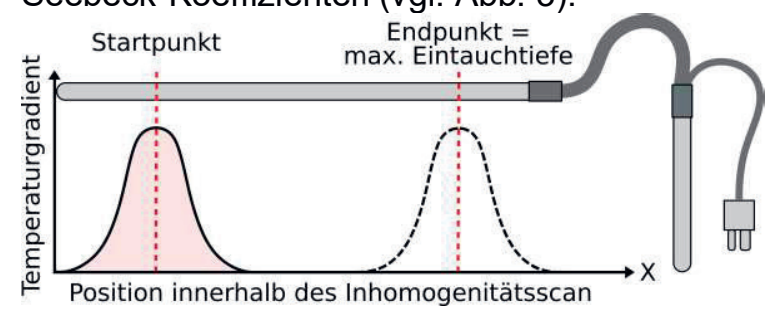

\section{Abb. 3: Verschiebung des Temperatur- gradienten innerhalb des Ein- Gradienten-Verfahrens}

Um die Veränderung des lokalen SeebeckKoeffizienten entlang des Thermoelementes dabei möglichst hoch aufzulösen, muss ein schmaler Temperaturgradient entlang des Sensors verschoben werden. Die Breite des Temperaturgradienten beschreibt dabei den Bereich, in dem die Variation des lokalen Seebeck-Koeffizienten erfasst werden kann. Je schmaler der Gradient ist, desto mehr Thermospannung resultiert aus einem kurzen Teilbereich des Thermoelementes. Lokal stark begrenzte Inhomogenitäten werden in breiten Temperaturgradienten nur in Verbindung mit homogenen Teilbereichen des Thermomaterials erfasst. Wird der Temperaturgradient daraufhin geschärft, verbessert sich die räumliche Auflösung des Scanners soweit bis der inhomogene Teilabschnitt des Thermoelementes vollständig erfasst wird.

Um eine geeignete Versuchsanlage zur Bestimmung der Inhomogenität zu entwickeln, muss der Temperaturgradient messtechnisch erfasst und dessen Breite rechnerisch 
bestimmt werden. Nach der von Webster et al. [4] beschriebenen Vorgehensweise kann die maximale Auflösung für die nachfolgend vermessenen Typ $S$ Thermoelemente mit $6 \mathrm{~mm}$ Keramikschutzrohr anhand des Temperaturverlaufs in der Versuchsanlage bestimmt werden. Dabei wird der Temperaturverlauf im Inhomogenitätsscanner unter Berücksichtigung der Wärmeleitung erfasst und in den wirkenden Gradienten umgewandelt. Der Temperaturgradient kann mathematisch durch die Ableitung des Temperaturverlaufs entlang des Thermoelementes bestimmt werden.

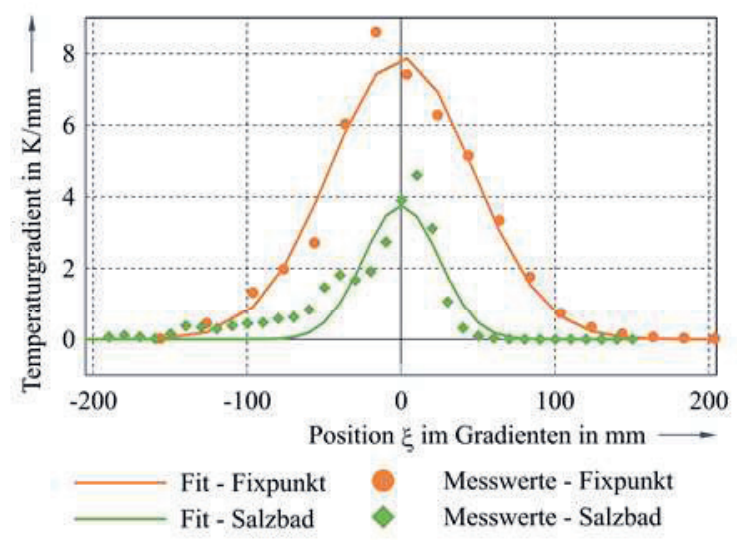

Abb. 4: Vergleich der Temperaturgradienten realer Ein-Gradienten-Scanner aufgenommen mit Typ $S$ Thermoelement in $6 \mathrm{~mm}$ Schutzkeramik; Approximation über Gaußfunktion

In Abbildung 4 sind die vorherrschenden Temperaturgradienten in zwei typischen Versuchsanlagen zur Ein-GradientenInhomogenitätsmessung gegenübergestellt.

Die Halbwertsbreite der Gauß-Fit-Funktion beschreibt dabei nach Hill et al. [5] die maximale räumliche Auflösung des Scanners. Diese lag bei den verwendeten Prüflingen bei $25 \mathrm{~mm}$ im Salz-Kalibrierbad und $46 \mathrm{~mm}$ im Silber-Fixpunkt. Aufgrund der besseren räumlichen Auflösung kann das Kalibrierbad, im Vergleich zu dem verwendeten Fixpunkt, auch deutlich kleinere Inhomogenitäten erfassen.

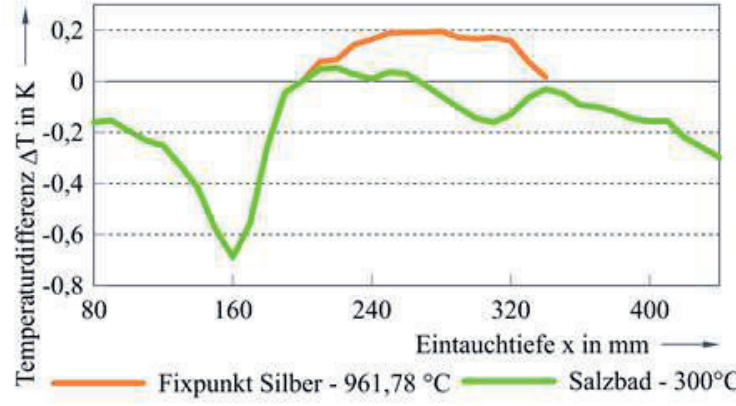

Abb. 5: Vergleich der Inhomogenitätsprofile eines Typ $S$ Thermoelementes in $6 \mathrm{~mm}$ Schutzkeramik; Aufgenommen in verschiedenen Versuchsanlagen

Aus den Vergleichen verschiedener Kalibriereinrichtungen (in Abb. 4 und 5) geht hervor, dass sich vor allem Salz- und ÖlKalibrierbäder zum Vermessen der Sensoren im Ein-Gradienten-Verfahren eignen.

Die direkten Auswirkungen der Gradientenbreite auf die erfassten Inhomogenitäten lassen sich in Abb. 5 erkennen. Die Untersuchung desselben Thermoelementes in mehreren Versuchsanlagen weist dabei deutliche Unterschiede im erfassten Inhomogenitätsprofil auf. Die lokal ausgeprägten Inhomogenitäten des vermessenen Thermoelementes werden hier nur vom Gradienten des Kalibrierbades erfasst. In der Silber-Fixpunktzelle werden diese lokal begrenzten Inhomogenitäten aufgrund des breiten Temperaturgradienten und des begrenzten Bauraums nicht erfasst (vgl. Abb. 5).

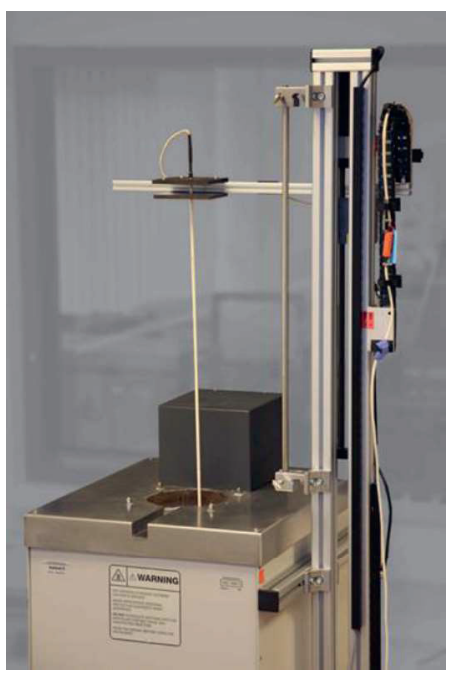

Abb. 6: Konstruktive Umsetzung des EinGradienten-Inhomogenitätsscans am Salz-Kalibrierbad 
Für die Untersuchungen der Homogenität von Thermoelementen bietet sich im Rahmen der verfügbaren Kalibrieranlagen ein SalzKalibrierbad mit großer Eintauchtiefe an (vgl. Abb. 6). Dieses wird zur automatisierten Umsetzung der Inhomogenitätsscans mit einer Linearachse und einer erweiterten Auswertesoftware ausgestattet.

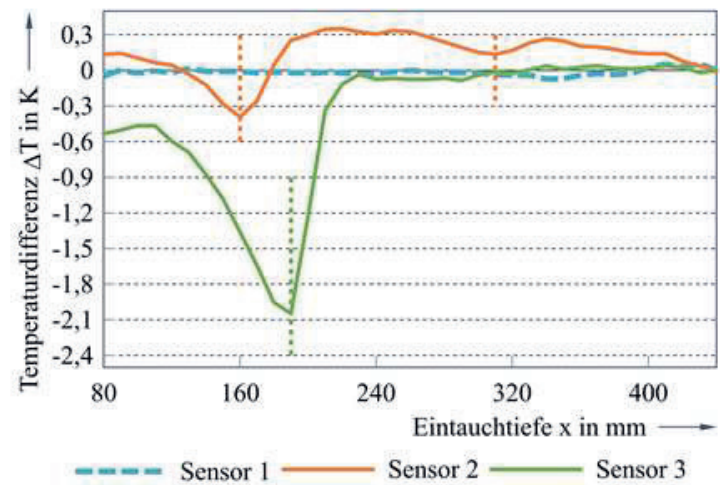

Abb. 7: Erfasste Inhomogenitäten diverser Typ S Thermoelemente mit $6 \mathrm{~mm}$ Schutzkeramik im Salz-Kalibrierbad bei $300{ }^{\circ} \mathrm{C}$

Über den vorgestellten Ein-GradientenScanner wird die Aufnahme der Thermoelemente bis zu einer Länge von $440 \mathrm{~mm}$ möglich.

In Abbildung 7 sind die untersuchten Typ S Thermoelemente gegenübergestellt. Sensor 2 und 3 sind dabei ein exemplarisches Beispiel, welche Messfehler Inhomogenitäten im realen Betrieb erzeugen können. Die auf den Endwert bei maximaler Eintauchtiefe normierten Inhomogenitätsprofile zeigen Abweichungen von bis zu 2,07 K.

\section{Messungen im Zwei-Gradienten-Verfahren}

Beim Zwei-Gradienten-Verfahren wird das Thermoelement mit einer lokal begrenzten Hitzequelle überstrichen, während die Messund Vergleichsstelle auf einer konstanten Temperatur gehalten werden. Dadurch entstehen zwei gegenläufige Gradienten auf dem Thermoelement (vgl. Abb. 8) [3].

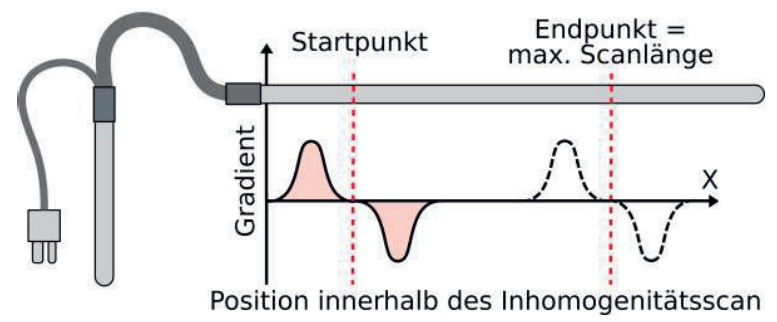

Abb. 8: Verschiebung der Temperaturgradienten innerhalb des ZweiGradienten-Verfahrens
In den beiden gegenläufigen Gradienten entsteht jeweils eine Teil-Thermospannung. Bei einem homogenen Thermoelement sind die Thermospannungen in beiden Gradienten gleich groß, die gemessene Thermospannung beim Überfahren des Sensors ist gleich null. Während der Messung eines inhomogenen Thermoelementes sorgt der Unterschied zwischen den lokalen Seebeck-Koeffizienten in beiden Gradienten für eine von null abweichende Thermospannung. Diese Thermospannung ist dabei ein Maß für den Unterschied der beiden lokalen SeebeckKoeffizienten innerhalb der gegenläufigen Gradienten. Dementsprechend kann die erfasste Thermospannung auch nicht direkt in eine äquivalente Temperatur umgerechnet werden.



Abb. 9: Nahaufnahme des Zwei-GradientenScanners

Um einen Zwei-Gradienten Scan durchführen zu können, wird eine räumlich stark begrenzte Hitzequelle mit einem klar definierten Temperaturfeld benötigt. Dieses kann beispielsweise durch einen nach außen isolierten Widerstandsheizer mit zusätzlicher Luftkühlung zur Schärfung der Gradienten erzeugt werden. Um automatisierte Inhomogenitätsprofile analog zum EinGradienten-Verfahren aufnehmen zu können, wird dieser an einer geeigneten Lineareinheit angebracht und über das zu untersuchende Thermoelement gefahren (vgl. Abb. 9). Der untersuchte Sensor wird hingegen in der Messeinrichtung fixiert. 


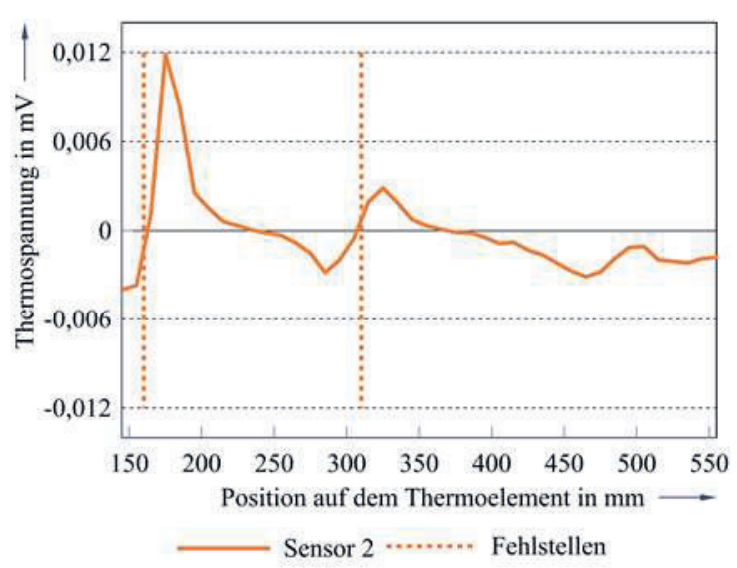

Abb. 10: Erfasstes Inhomogenitätsprofil des Sensors 2 (Typ $S$ mit $6 \mathrm{~mm}$ Schutzkeramik) im Zwei-GradientenVerfahren bei $300{ }^{\circ} \mathrm{C}$

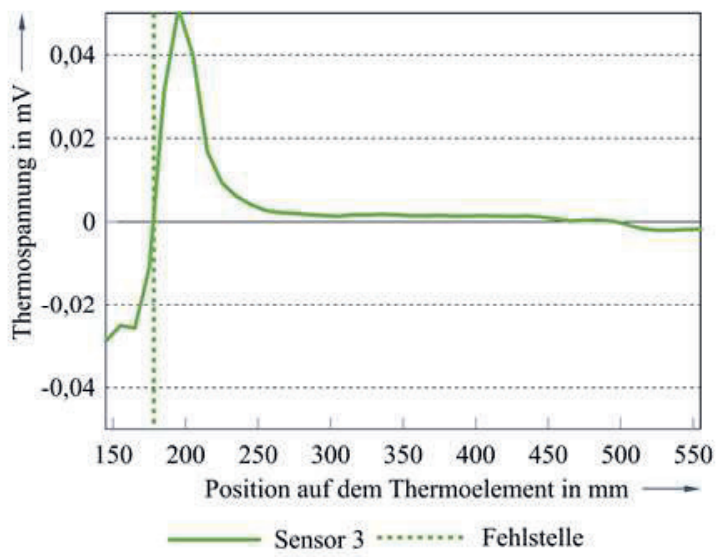

Abb. 11: Erfasstes Inhomogenitätsprofil des Sensors 3 (Typ $S$ mit $6 \mathrm{~mm}$ Schutzkeramik) im Zwei-GradientenVerfahren bei $300{ }^{\circ} \mathrm{C}$

Aufgrund der zwei gegenläufigen Gradienten folgt bei diesem Versuchsaufbau ein vollkommen unterschiedlicher Verlauf zu der im Ein-Gradienten-Verfahren erfassten Thermospannung. Einzelne Fehlstellen äußern sich im Zwei-Gradienten-Scan durch zwei charakteristische Peaks und einen Nulldurchgang im Inhomogenitätsprofil. (vgl. Abb.10 und 11).

Dieser charakteristische Verlauf resultiert einzig aus den gegenläufigen Temperaturgradienten. Wenn eine Inhomogenität im ZweiGradienten-Verfahren erfasst wird, so ändert sich erst die Teil-Thermospannung im ersten Gradienten. Anschließend erreicht die Inhomogenität die Mitte der Hitzequelle und trägt nicht zur erfassten Thermospannung bei. Erst beim Verlassen des Heizers wird die Inhomogenität wieder erfasst und erzeugt im gegenlaufenden Gradienten einen zweiten, umgekehrten Peak im Inhomogenitätsprofil. Die anhand der ausgeprägten Verläufe im Inhomogenitätsprofil erfassten Fehlstellen der Sensoren 2 und 3 korrelieren dabei mit den Messungen im Ein-Gradienten-Verfahren (vgl. Abb. 7, 10 und 11).

Die weitere Auswertung der erfassten Inhomogenitätsprofile ist hingegen schwierig, da die hier erfasste Thermospannung keine direkte Aussage über die tatsächlich ausgelösten Messfehler liefert. Um den realen Anwendungsfall eines Thermoelementes (näherungsweise in einem Gradienten) nachzuvollziehen, muss der hier erfasste Unterschied zwischen den SeebeckKoeffizienten beider Gradienten umgerechnet werden. Eine direkte Umrechnung der Ein- und Zwei-Gradienten-Verfahren nach bekannten Methoden konnte, trotz vergleichbarer Temperaturgradienten und Absoluttemperaturen in beiden Versuchsanlagen, bei den bisher untersuchten Thermoelementen nicht erreicht werden.

\section{Vergleich der verwendeten Versuchsanlagen und Messverfahren}

Aus den entwickelten Verfahren und den Untersuchungen der Homogenität verschiedener Edelmetall-Thermoelemente gehen die jeweiligen Anwendungsbereiche der beiden Funktionsprinzipe hervor.

Dabei eignet sich der Ein-GradientenInhomogenitätsscan aufgrund seines Temperaturverlaufs entlang des Thermoelementes für direkte Aussagen über den lokalen Seebeck-Koeffizienten des Thermomaterials im Temperaturgradienten. In Abhängigkeit von der gewählten Einrichtung zur Erzeugung der homogenen Zone wird die zu untersuchende Länge des Thermoelementes jedoch stark beschränkt. Des Weiteren wird in der Regel eine zusätzliche Kalibriereinrichtung mit hohen Anforderungen an die räumliche Homogenität der heißen Zone nötig.

Das Zwei-Gradienten-Verfahren zeichnet sich vor allem durch die nahezu unbegrenzte Länge des zu untersuchenden Sensors aus. Der Einsatzbereich des Zwei-Gradienten-Scanners wird dabei jedoch durch die Entstehung der Thermospannung in den gegenläufigen Gradienten begrenzt. Hier ist ohne weitere Anstrengungen keine direkte Aussage über die Ausprägung des lokalen SeebeckKoeffizienten anhand der erfassten Thermospannung möglich. 
Die Vergleichbarkeit der ermittelten Inhomogenitätsprofile ist jedoch unter gewissen Umständen gegeben. Wenn anstatt dem absoluten Betrag der Inhomogenität die Existenz und die Lage der Fehlstellen nachgewiesen werden soll, liefern Ein- und Zwei-Gradienten-Verfahren vergleichbare Ergebnisse. Die vollständige Beschreibung der ausgeprägten Inhomogenitäten wird aktuell jedoch nur im Ein-Gradienten-Verfahren möglich.

\section{Literaturnachweis}

[1] F. Bernhard, Handbuch der Technischen Temperaturmessung, 873 (2014); doi: 10.1007/978-3-642-24506-0

[2] Calibration Guide EURAMET cg-8, Calibration of thermocouples, Version 2.1, EURAMET e.V. Braunschweig, Oktober 2011

[3] M. Holmsten, J. Ivarson, R. Falk, M. Lisbeck, L.E. Josefson, Inhomogeneity Measurements of Long Thermocouples using a Short Movable Heating Zone, Int. Journal of Thermophysics, (2008); doi: 10.1007/s10765-008-0418-9

[4] E. Webster, D. R. White, Thermocouple homogeneity scanning, Metrologia 52 (2015); doi: 10.1088/0026-1394/52/1/130

[5] K. D. Hill, D. J. Gee, Quantifying the Calibration Uncertainity Attributable to Thermocouple Inhomogeneity, AIP Conference Proceedings 1552, 520 (2013); doi: 10.1063/1.4819595 